\title{
Mapping human serum-induced gene networks as a basis for the creation of biomimetic periosteum for bone repair
}

\author{
Rawiya Al Hosni ${ }^{1}$, Mittal Shah ${ }^{1}$, Umber Cheema $^{1}$, Helen C. Roberts ${ }^{2}$, Frank P. Luyten ${ }^{3}$, \\ Scott J. Roberts ${ }^{1,3,4, *}$ \\ ${ }^{1}$ Department of Materials and Tissue, Institute of Orthopaedics and Musculoskeletal Science, University College London, Stanmore, UK \\ ${ }^{2}$ Department of Natural Sciences, Faculty of Science \& Technology, Middlesex University, London, UK \\ ${ }^{3}$ Skeletal Biology and Tissue Engineering Centre, Department of Development and Regeneration, KU Leuven, Leuven, Belgium and \\ ${ }^{4}$ Department of Comparative Biomedical Sciences, The Royal Veterinary College, London, UK
}

\section{A R T I C L E I N F O}

\section{Article History:}

Received 5 December 2019

Accepted 23 March 2020

Available online xxx

\section{Keywords:}

periosteum

bone defect

periosteal cells

transcriptomics

cell potency

\begin{abstract}
A B S T R A C T
Background: The periosteum is a highly vascularized, collagen-rich tissue that plays a crucial role in directing bone repair. This is orchestrated primarily by its resident progenitor cell population. Indeed, preservation of periosteum integrity is critical for bone healing. Cells extracted from the periosteum retain their osteochondrogenic properties and as such are a promising basis for tissue engineering strategies for the repair of bone defects. However, the culture expansion conditions and the way in which the cells are reintroduced to the defect site are critical aspects of successful translation. Indeed, expansion in human serum and implantation on biomimetic materials has previously been shown to improve in vivo bone formation.

Aim: This study aimed to develop a protocol to allow for the expansion of human periosteum derived cells (hPDCs) in a biomimetic periosteal-like environment.

Methods: The expansion conditions were defined through the investigation of the bioactive cues involved in augmenting hPDC proliferative and multipotency characteristics, based on transcriptomic analysis of cells cultured in human serum.

Results: Master regulators of transcriptional networks were identified, and an optimized periosteum-derived growth factor cocktail (PD-GFC; containing $\beta$-estradiol, FGF2, TNF $\alpha$, TGF $\beta$, IGF-1 and PDGF-BB) was generated. Expansion of hPDCs in PD-GFC resulted in serum mimicry with regard to the cell morphology, proliferative capacity and chondrogenic differentiation. When incorporated into a three-dimensional collagen type 1 matrix and cultured in PD-GFC, the hPDCs migrated to the surface that represented the matrix topography of the periosteum cambium layer. Furthermore, gene expression analysis revealed a down-regulated WNT and TGF $\beta$ signature and an up-regulation of CREB, which may indicate the hPDCs are recreating their progenitor cell signature.

Conclusion: This study highlights the first stage in the development of a biomimetic periosteum, which may have applications in bone repair.
\end{abstract}

(c) 2020 International Society for Cell and Gene Therapy. Published by Elsevier Inc. All rights reserved.

\section{Introduction}

The periosteum is a highly vascularized connective tissue that covers bone. It is composed of two layers: an inner cambium layer that contains a skeletal stem cell population and an outer fibrous layer composed of fibroblasts embedded within a collagen type 1 matrix [1-3]. Collagen fibers located in the periosteum are relatively small and compact compared with collagen bundles found in the skin [3]. The periosteum stem cell population located in the cambium layer acts as a major participant in bone development and fracture healing [4,5]. This population of multipotent cells are capable of self-

\footnotetext{
* Corresponding author: Dr. Scott J. Roberts, Department of Comparative Biomedical Sciences, The Royal Veterinary College, London, UK.

E-mail address: sjroberts@rvc.ac.uk (S.J. Roberts).
}

renewing and differentiating into osteogenic and chondrogenic lineages, which is partly dependent on the mechanical stabilization of the fracture $[4,6]$. During endochondral ossification, for non-stabilized fracture repair, the periosteum contributes up to $90 \%$ of the chondrogenic cells required for the early cartilaginous callus [7].

Bone has an extraordinary capacity for repair; however, repair fails when the defect reaches a critical size and/or becomes a non-union [8]. These bone defects are typically in compromised conditions such as tumors, infection, major trauma or congenital malformation resulting in significant disruption of the periosteum layer [9]. Various surgical procedures have been implemented to overcome failed healing including the use of autologous bone, which is the current gold standard. Additionally, generation of pseudo-periosteum using the Masquelet membrane technique and periosteum grafts have shown potential for bone repair [10]. Despite surgical advances, there is an unmet medical need 
for an "off-the-shelf" regenerative solution for large bone defects. This would overcome many of the caveats associated with surgery, including donor site morbidity, risk of infection and discomfort [4]. To overcome these issues, regenerative medicine aims to repair damaged tissue using cell-based therapies.

Periosteum-derived cells (PDCs) are a promising cell source for bone repair, predominantly due to their intrinsic bone forming capacity $[11,12]$. Indeed, this cell population displays multipotent characteristics at the single cell level and the ability to form a bone ossicle with hematopoietic compartment in vivo [13,14]. However, cell potency is lost during expansion over long periods of time, and hence there is a limited window to apply these cells to clinical strategies [15]. This is similar to other adult stem cell populations, and as such there has been a considerable effort to enhance the proliferative and differentiation capacity through optimization of culture conditions. Currently, human mesenchymal stromal cells (hMSCs) are generally expanded in conventional culture media using fetal bovine serum (FBS), which provides bioactive molecules such as growth factors, hormones and proteins necessary for cell growth and function [15]. However, a myriad of disadvantages and limitations are associated with its use. Indeed, it is an ill-defined supplement and is inconsistent with regard to the quality and quantity of bioactive compounds present, resulting in lot-to-lot variability [16,17]. Additionally, the use of a xenogenic serum could result in possible immune-mediated rejection if used for clinical translation of cell therapies [18]. Furthermore, ethical concerns associated with the harvesting of FBS from fetal calves presents another significant challenge to its use [19].

Various serum-free and xeno-free media have been developed in an attempt to negate the potential variability and safety concerns associated with the use of animal serum in clinical applications [20]. Indeed, we have reported the beneficial effect of human allogeneic serum (hAS) on the in vitro differentiation and expansion of human PDCs (hPDCs). These findings were translated successfully in vivo where cells expanded in hAS outperformed FBS expanded cells in bone-forming capacity, thus demonstrating culture memory from in vitro expansion [11]. Interestingly, platelet lysate has also been shown to increase hPDC bone-forming capacity [21]. Nevertheless, the use of hAS for clinical applications would require extensive screening and a well-characterized pool of donors, which could prove problematic in a clinical setting. Therefore, it is attractive to develop alternative methods of incorporating the necessary factors required to maintain the proliferative and progenitor-like characteristics of hPDCs. Furthermore, an appropriate scaffold that replicates the periosteum and allows the creation of a suitable delivery device is required for clinical translation.

Herein, a protocol for the expansion of hPDCs in a biomimetic periosteal-like environment to facilitate delivery to a bone defect is proposed. The expansion conditions were defined through the transcriptomic analysis of cells cultured in hAS. Gene network analysis was utilized for the identification of the key master regulators activated by hAS-culture. Through the identification and careful selection of these growth factors, we were able to achieve serum mimicry with regard to cell morphology, proliferative capacity and chondrogenic differentiation. When cells were incorporated into a three-dimensional (3D) collagen type 1-rich matrix, the periosteum-derived growth factor cocktail (PD-GFC) caused the hPDCs to migrate to the surface representing the matrix topography of the periosteum cambium layer. Furthermore, gene expression analysis revealed down-regulated WNT and TGF $\beta$ with up-regulated CREB signaling, which may indicate that the hPDCs are re-creating their progenitor cell signature. The successful development of this cell-specific cocktail is the first step in developing a periosteum-like environment that mimics the biology of the periosteum.

\section{Methods}

\section{Human periosteum-derived cell cultures}

hPDCs were isolated from biopsies obtained from patients undergoing orthopedic surgery as described elsewhere [13,14]. Human
Medical Research (KU Leuven) approved all procedures, and the patient-informed consent forms were obtained. Subsequently, hPDCs from a pool of six donors (age $=14.9 \pm 2.1$ years; male:female $=4: 2$ ) were expanded in growth medium (GM) consisting of high-glucose Dulbecco's Modified Eagle's Medium (DMEM; Invitrogen, Paisley, UK) supplemented with $10 \%$ batch-tested FBS and antibiotic-antimycotic solution (100 units/mL penicillin, $100 \mu \mathrm{g} / \mathrm{mL}$ streptomycin and 0.25 $\mu \mathrm{g} / \mathrm{mL}$ amphotericin $\mathrm{B}$; Invitrogen) to passage 5 . All experiments described herein were performed in pooled hPDCs at passage 6 . We have previously published the CD marker profile (CD73+, CD90+ and CD105+), tri-linage differentiation capacity (osteogenic, chondrogenic and, adipogenic) and in vivo bone-forming capacity (bone ossicle with marrow) of this donor pooled population [11,13].

\section{Selection of growth factors by RNA sequencing}

We have previously tested the three hAS serum pools (minimum of 15 donors) used herein, with two hPDC cell pools and observed very similar growth characteristics with each combination. This data is reported in Roberts et al. [11]. To analyze the effects of the culture systems on the hPDCs, RNA sequencing (RNAseq) was performed on the aforementioned hPDC pool at sub-confluence (initial seeding density 1000 cells $/ \mathrm{cm}^{2}$ ) following culture for 6 days in hAS serum ( $n=3$; donor pooled) or FBS ( $n=3$; different Gibco batch). RNA was extracted using the Illumina TruSeq Standard Total RNA Sample Prep Kit (Illumina, San Diego, CA, USA). RNA integrity was validated using a BioAnalyzer (Agilent, Santa Clara, CA, USA).

RNAseq was performed at the Nucleomics Core (KU Leuven, Belgium). Libraries were generated from $2 \mu \mathrm{g}$ RNA using the TruSeq library prep kit (Illumina) as per the manufacturer's recommendations. Sequencing of all samples was carried out on the HiSeq2000 (Illumina), with read lengths of 50 base pairs. Between 25.0 and 39.3 million reads were sequenced for each sample. Distributions of the average read quality was calculated using the ShortRead 1.18.0 package from Bioconductor (http://www.bioconductor.org). Base calling accuracy, measured by the Phred quality score, was measured in all samples, with values of $\geq 30$.

Pre-processed reads were aligned with the reference genome of Homo sapiens (GRCh37.73) using Tophat v2.0.8b. Quality filtering to remove reads from the alignment that were non-primary mappings or had a mapping quality $\leq 20$ was performed with SAMtools 0.1.19. The number of mapped reads varied between 17.7 and 28.3 million reads per sample. Subsequently, Cufflinks v2.1.1 was used to extract unique transcript-related features. A list of gene-level coordinates was constructed by merging the exon chains of transcripts that belong to the same gene using mergeBed from the Bedtools v2.17.0 toolkit. The number of exons and number of transcripts was subsequently computed for each gene. Genes where reads could be attributed to more than one gene (ambiguous) or could not be attributed to any gene (no feature) or for which all samples had less than 1 count-per-million (absent) were removed. This left 15518 identified genes. GC-content was corrected in each sample using full quantile normalization on bins of GC-content with the EDASeq package from Bioconductor. Between-sample normalization (sample-specific variation due to the library size and RNA composition) were corrected for using full quantile normalization with the EDASeq package from Bioconductor. A relative log expression plot using $\log _{2}$-scale normalized counts for each gene as expression level was constructed and resultant values of close to 0 indicated the normalization was successful. For each gene, the expression levels of both conditions were estimated from the raw counts with the DESeq 1.14.0 package of Bioconductor and tested for differential expression based on a model using the negative binomial distribution. To select genes the corrected $P$ value was set at $<0.05$, which resulted in 1331 differentially regulated genes $\left(\log _{2}\right.$ ratio $\leq-1$ or $\log _{2}$ ratio $\left.\geq 1\right)$. 
The dataset was validated using quantitative polymerase chain reaction (qPCR; described subsequently). Differentially regulated genes were selected based on fold-change ratios from the RNAseq datasets between cells cultured in hAS compared with FBS. The genes selected for data validation demonstrated a high up- or down-regulated expression (fold change $>20$ ), moderate change (fold change 10-20) and minimal up- or down-regulation (fold change $<10$ ) (gene panel detailed in supplementary Figure 1). The genes that were significantly differentially expressed between hAS and FBS were initially interrogated through the online platform DAVID (Database for Annotation, Visualization and integrated Discovery, http://david.abcc.nciforf.gov/) to determine the prominent biological processes involved in each of these culture conditions. The data were further analyzed using Ingenuity Pathway Analysis (IPA) (Qiagen, Venlo, the Netherlands) to identify activated networks and upstream regulators controlling the divergent biology observed between hAS and FBS. All genes demonstrating a fold change $>2$ with respect to expression in hAS compared with FBS with a $P$ value $<0.05$ were extracted from the RNAseq data for analysis with IPA. Associated genomic relationships were generated including regulators indirectly connected to the primary targets observed, to help identify the upstream regulators. Factors were selected according to their relationships with 10 gene networks. Factors identified as significant upstream regulators were mapped onto the gene networks to interrogate their function in respect to each of the identified processes. A literature search was performed to determine any precedent for each factor in mesenchymal cell expansion/culture and to select appropriate concentrations for further investigation.

\section{"Leave-one-factor-out" strategy}

Upstream regulators identified via IPA were assessed in vitro for their potential role in controlling hPDC gene expression, proliferation and metabolic activity. The selected factors (vascular endothelial growth factor $-\alpha$ [VEGF $\alpha ; 10 \mathrm{ng} / \mathrm{mL}$, Peprotech, London, UK), dexamethasone $\left(1 \times 10^{-8} \mathrm{~mol} / \mathrm{L}\right.$, Sigma, Gillingham, UK), Wnt3A (10 ng/mL, R\&D Systems, Minneapolis, MN, USA), $17-\beta$-estradiol $\left(1 \times 10^{-9} \mathrm{~mol} / \mathrm{L}\right.$, Sigma $)$, fibroblast growth factor-2 (FGF2; $10 \mathrm{ng} / \mathrm{mL}$, Thermo Fisher Scientific, Paisley, UK), tumor necrosis factor $-\alpha$ (TNF $\alpha ; 5 \mathrm{ng} / \mathrm{mL}$, Peprotech), transforming growth factor (TGF $\beta ; 10 \mathrm{ng} / \mathrm{mL}$, Peprotech), insulin growth factor-1 (IGF-1; $20 \mathrm{ng} / \mathrm{mL}$, Peprotech) and platelet derived growth factor (PDGF-BB; $10 \mathrm{ng} / \mathrm{mL}$, Peprotech) were applied in a "leave-one-factor-out" strategy to identify the key regulators involved in stimulating the cells' proliferation and differentiation in vitro. hPDCs were seeded on a 24-well plate at a density of $1000 \mathrm{cells} / \mathrm{cm}^{2}$ and cultured for 7 days in the varying factor conditions supplemented with $1 \%$ FBS (concentration that when in combination with additional defined serum-free replacements the culture becomes notably defined [22]) in phenol red free, high-glucose DMEM (Thermo Fisher Scientific). Factors critical to analyzed cell responses were combined to create PD-GFC.

\section{Analysis of in vitro cell response}

To assess metabolic changes the cells were also treated with PrestoBlue Cell Viability Reagent (Thermo Fisher Scientific) following manufacturer's instructions. This resazurin-based assay induces changes in absorbance at $600 \mathrm{~nm}$, which were recorded using a Tecan Infinite 200 PRO plate reader (Tecan, Männedorf, Switzerland). Double-stranded DNA (dsDNA) quantification was carried out using the Qubit dsDNA High Sensitivity Assay Kit (Thermo Fisher Scientific) following manufacturer's instructions. Briefly, cell monolayers were lysed in RLT buffer (Qiagen) plus $10 \mu \mathrm{L} / \mathrm{mL} \beta$-mercaptoethanol (Sigma) [23]. The lysate was diluted $1 / 10$ in nuclease-free water (Sigma). Readings were obtained using the Qubit 3.0 fluorometer (Thermo Fisher Scientific) and were calculated as total dsDNA [ng/ $\mu \mathrm{L}$ ]. Cell spreading was analyzed by culturing cells for 7 days at a cell density of 1000 cells $/ \mathrm{cm}^{2}$, which were subsequently fixed with $10 \%$ neutral buffered formalin (NBF) (Sigma). Actin cytoskeleton was visualized through staining with phalloidin and nuclei counterstained with 4',6-diamidino-2-phenylindole (Sigma). Cell spreading was calculated via a width-to-length ratio measured digitally using Image (National Institutes of Health, Bethesda, MD, USA).

\section{Quantitative PCR}

Total RNA was isolated using the RNeasy kit (Qiagen) following manufacturer's instructions. RNA was quantified using a NanoDrop spectrophotometer (Thermo Fisher Scientific) measuring at 260/ $280 \mathrm{~nm}$, and $1 \mu \mathrm{g} \mathrm{RNA} /$ sample was reverse transcribed using the High Capacity cDNA Reverse Transcriptase Kit (Thermo Fisher Scientific) with the program: $25^{\circ} \mathrm{C}$ for $10 \mathrm{~min}, 37^{\circ} \mathrm{C}$ for $120 \mathrm{~min}, 85^{\circ} \mathrm{C}$ for 5 min and infinite hold at $4^{\circ} \mathrm{C}$. Transcribed cDNA was assessed via the CFX96 Touch Real-Time PCR Detection System (40 cycles) using the iTaq Universal SYBR Green Supermix (Bio-Rad, Hertfordshire, UK). Primers were designed using Primer3 (listed in supplementary Table I) and were designed to span an intron to isolate RNA specific amplification. Relative differences in expression were calculated using $2^{-\Delta \Delta \mathrm{Ct}}[24]$ normalized to HPRT expression.

\section{Analysis of hPDC growth kinetics}

The effect of PD-GFC on hPDC characteristics was assessed by expanding the cells in either PD-GFC medium or conventional GM. Cells were initially grown in conventional GM containing $10 \%$ or $1 \%$ FBS until confluency was reached and further subcultured in either GM (containing either 1\% FBS or 10\% FBS) or PD-GFC medium over four passages $(n=3)$. Cumulative population doubling was calculated using the following equation: $\mathrm{PDL}=3.32(\log \mathrm{Xe}-\log \mathrm{Xb})+\mathrm{S}$, where $\mathrm{Xb}$ is the cell number at the beginning of the culture, $\mathrm{Xe}$ is the cell number at the end of the culture and $S$ is the number of population doublings at the start of the culture.

\section{Analysis of hPDC differentiation}

Previously expanded cells (in either $10 \%$ FBS or PD-GFC) were subcultured at $70 \%$ confluency and then assessed for their differentiation capacity. Osteogenic differentiation was assessed using a defined osteogenic growth factor cocktail [46] on hPDCs passaged twice in either GM or PD-GFC- seeded at a density of 3000 cells $/ \mathrm{cm}^{2}$ in their initial growth mediums for $24 \mathrm{~h}$. The medium was replaced with conventional GM supplemented with ascorbate-2-phosphate $(57 \mu \mathrm{mol} / \mathrm{L}$, Sigma), TGF- $\beta 1$ (10 ng/mL, Peprotech), epidermal growth factor (EGF; $20 \mathrm{ng} / \mathrm{mL}$, Invitrogen), interleukin-6 (IL-6; $10 \mathrm{ng} / \mathrm{mL}$, Peprotech), calcium ( $3 \mathrm{mmol} / \mathrm{L}$, Sigma) and phosphate ( $2 \mathrm{mmol} / \mathrm{L}$, Sigma). The cells were differentiated for a period of 7 days with media changed every $48 \mathrm{~h}$. Differentiation was assessed through staining with alizarin red solution ( $\mathrm{pH} 4.2$, Sigma). Quantification of calcium mineral deposits was performed by dissolving the incorporated dye with $10 \%$ cetylpyridinium chloride (Sigma) for $60 \mathrm{~min}$ at room temperature. Absorbance was measured spectrophotometrically at $570 \mathrm{~nm}$.

Chondrogenic differentiation was assessed on hPDCs expanded over three passages in either GM or PD-GFC and further seeded in high-density micromasses at a cell density of 5000 cells $/ \mu \mathrm{L}$. Cells were allowed to adhere before culturing in chondrogenic medium consisting of low-glucose DMEM (Invitrogen), 1x insulin-transferrinselenium supplement (Sigma), dexamethasone (100 nmol/L, Sigma), Y27632 (10 $\mu \mathrm{mol} / \mathrm{L}$, Axon Medchem, Groningen, Netherlands), ascorbic acid (50 $\mu \mathrm{g} / \mathrm{mL}$, Sigma), proline (40 $\mu \mathrm{g} / \mathrm{mL}$, Sigma) and TGF- $\beta 1$ (10 ng/mL, Peprotech) for 7 days. Differentiation was evaluated by staining with Alcian Blue ( $\mathrm{pH}$ 2.0, Sigma) overnight at room temperature. Quantification of proteoglycans was carried out by extracting the Alcian Blue dye from the micromass using $6 \mathrm{~mol} / \mathrm{L}$ guanidine hydrochloride (Sigma) and measuring the absorbance at $620 \mathrm{~nm}$. 
Adipogenic differentiation was assessed following hPDC culture over two passages in either GM or PD-GFC at a cell density of 30000 cells/well in a 48-well plate for a period of 21 days. The cells were seeded in GM until the cells reached confluency after $48 \mathrm{~h}$. Once confluency was reached the media was substituted with adipogenic differentiation media containing $10 \% \mathrm{FBS}$, insulin ( $1 \mu \mathrm{g} / \mathrm{mL}$, Sigma), dexamethasone (0.1 $\mu \mathrm{mol} / \mathrm{L}$, Sigma), isobutylmethylxanthine (IBMX; $4.5 \mu \mathrm{mol} / \mathrm{L}$, Sigma) and indomethacin (125 $\mu \mathrm{mol} / \mathrm{L}$, Sigma). Medium was changed every 2 days. Production of fat droplets was assessed using Oil Red $O$ stain (Sigma) and lipid droplet area quantified using ImageJ. Gene expression analysis was performed on stem, osteogenic, chondrogenic and adipogenic markers and qPCR conducted as previously described.

\section{Formation of collagen type 1 hPDC-seeded scaffold}

hPDCs were expanded until 80\% confluency was reached. Rat tail collagen type 1 (First Link,Wolverhampton, UK) gels were prepared according to the RAFT protocol. A collagen master-mix was prepared containing 10\% 10x DMEM, 80\% rat tail collagen type $1(2.05 \mathrm{mg} / \mathrm{mL}$ in $0.6 \%$ acetic acid) and a $10 \%$ neutralizing agent composed of $10 \mathrm{~mol} /$ $\mathrm{L} \mathrm{NaOH}$ and Hepes buffer (Thermo Fisher Scientific) [25]. The collagen solution was set on ice for a minimum of $30 \mathrm{~min}$ to allow for the movement of air bubbles out of the solution. The cells were prepared and incorporated into the collagen master mix to produce collagen scaffolds with a density of 100000 cells per gel. A volume of $1.3 \mathrm{~mL}$ of the collagen master mix was dispensed into 24 -well plates. The gels were allowed to gelate at $37^{\circ} \mathrm{C}$ for $15 \mathrm{~min}$, allowing for fibrillogenesis of the collagen hydrogel, comprising a network of intertwined fibrils with no inherent orientation consisting of a large excess fluid to collagen ratio [26]. To achieve a denser (10\%) collagen matrix, the gelated constructs underwent plastic compression using a hydrophilic RAFT absorber, following the Lonza RAFT 3D Cell Culture protocol. Briefly, the absorber was placed on top of the gel for 15 min to expel the fluid content through the main (basal) fluid leaving surface of the gel. This resulted in a $\sim 50$-fold increase in collagen density [27]. After plastic compression, the absorber was removed, and $1 \mathrm{~mL}$ of GM was added to the well. After $24 \mathrm{~h}$, the media were substituted with either $10 \%$ FBS-containing medium or the PD-GFC, and cultures were allowed to incubate for a period of 14 days. The collagen gels were subjected to histological analysis; paraffin-embedded sections were stained for cell cytoplasm and nuclei with hematoxylin and eosin, respectively.

\section{$R T^{2}$ Profiler PCR Array of human stem cell signaling}

To further interrogate the signaling mechanisms involved when the hPDCs were cultured in either FBS or PD-GFC in 3D, an $\mathrm{RT}^{2}$ Profiler PCR array (Qiagen) specific for stem cell-associated signaling pathways was utilized. The array profiles 84 key genes representative of six signal transduction pathways associated with identification, growth and differentiation of stem cells. The hPDCs were embedded within the collagen type 1 matrix as detailed earlier and subsequently cultured in either FBS-containing growth medium or PD-GFC for a period of 14 days. Samples were lysed using TRI reagent and RNA isolated using the chloroform phase separation technique and subsequently processed using the RNeasy Kit (Qiagen) according to manufacturer's instructions [28]. Single-strand cDNA was transcribed using $1 \mu \mathrm{g}$ total RNA, synthesized using the $\mathrm{RT}^{2}$ first strand kit (Qiagen). Real-time PCR was performed using the $\mathrm{RT}^{2}$ profiler array system according to manufacturer's instructions in combination with $\mathrm{RT}^{2}$ SYBR $^{\square}$ Green qPCR Mastermix (Qiagen) in a Bio-Rad CFX96 PCR system (Bio-Rad). Data analysis was conducted using a web-based $\mathrm{RT}^{2}$ PCR Profiler PCR array data analysis software (Qiagen). Genes were identified as up- or down-regulated with a fold change cutoff of 2 , with a corresponding $P$ value $<0.05$. $P$ values calculated based on
Student's $t$-test of the replicate $2^{-\Delta \Delta C t}$ values for each gene in the FBS group and corresponding PD-GFC experimental group.

\section{Stiffness of native periosteum and collagen type 1 scaffold}

Native bovine periosteum and seeded 10\% collagen type 1 cell laden scaffolds (following 14 days culture in PD-GFC; $n=3$ ) were washed with phosphate-buffered saline and embedded in optimal cutting temperature compound. Embedded samples were cryosectioned into $10-\mu \mathrm{m}$ sections, transferred onto glass slides and stored at room temperature. Atomic force microscopy (AFM) was performed on these samples using a JPK Nanowizard 1 AFM (JPK Instruments Ltd, Berlin, Germany) with RFESPA-75 cantilever $(\mathrm{k}=0.3 \mathrm{~N} / \mathrm{m})$. One hundred measurements were performed within a $10-\mu \mathrm{m}^{2}$ surface area in six locations of each tested region within the tissue/scaffold.

\section{Statistical analysis}

Data are expressed as mean \pm SEM. Statistical significance was determined using one-way analysis of variance with Fisher's least significant difference post hoc corrections applied or Student's $t$-test. Statistical significance is indicated on all graphs as follows: ${ }^{*} P<0.05$ ${ }^{* *} P<0.01,{ }^{* * *} P<0.001(\mathrm{n}=3)$. All statistical analysis was performed using GraphPad Prism version 6.0f for windows (GraphPad Prism Software, La Jolla, CA, USA, http://www.graphpad.com).

\section{Results}

\section{Identifying the regulators associated with hAS-induced hPDC potency}

hPDCs cultured in 10\% FBS and 10\% hAS for 6 days were subjected to RNASeq to analyse the transcriptomic profile of the cells in each condition. Before conducting further analysis with the data, the RNA Seq dataset was validated by qPCR profiling of the following markers: human transgelin 3 (TAGLN3), BCL2-related protein A1 (BCL2A1), sulfotransferase family 1B member 1 (SULT1B1), C-X-C motif chemokine ligand 22 (CXCL2), PDK1 family protein kinase (PDKA4), matrix metallopeptidase 1 (MMP1), semaphorin 3A (SEMA3A), angiopoietin-like 1 (ANGPTL1), platelet-derived growth factor subunit A (PDGFA), Noggin (NOG), alpha kinase 2 (ALPK2), cartilage intermediate layer protein 2 (CILP2), insulin like growth factor 1 (IGF1) and fibroblast growth factor 9 (FGF9). Relative expression values obtained using qPCR were comparable to those measured by RNA Seq with no significant differences observed (supplementary Figure 1).

To determine the specific factors involved in regulating the cell's characteristics when cultured in hAS relative to FBS, IPA software generated a list of all upstream regulators (factors that are predicted to have a downstream effect on other molecules) associated with multiple targets within each identified pathway (supplementary Table II). The upstream regulators were arranged with respect to their overlap $P$ value (right tail Fisher's exact test), which measures whether a statistically significant overlap is present between the experimental dataset and the genes regulated by the upstream transcriptional regulators. The regulators with a significant overlap are predicted to have a significant effect within the culture system. The top 20 upstream regulators were then recorded as potential factors responsible for the characteristics observed in cells cultured in hAS.

An additional methodology incorporated in the identification of key factors was the assessment of gene networks (supplementary Table III), whereby hub genes with direct and indirect gene-protein interactions were identified. To validate the top 20 upstream regulators, each was mapped to the network, and genes that identified as both upstream regulators and hub genes within these networks were recorded. Two networks associated with embryonic, organismal, tissue development and cellular movement, hematological system 

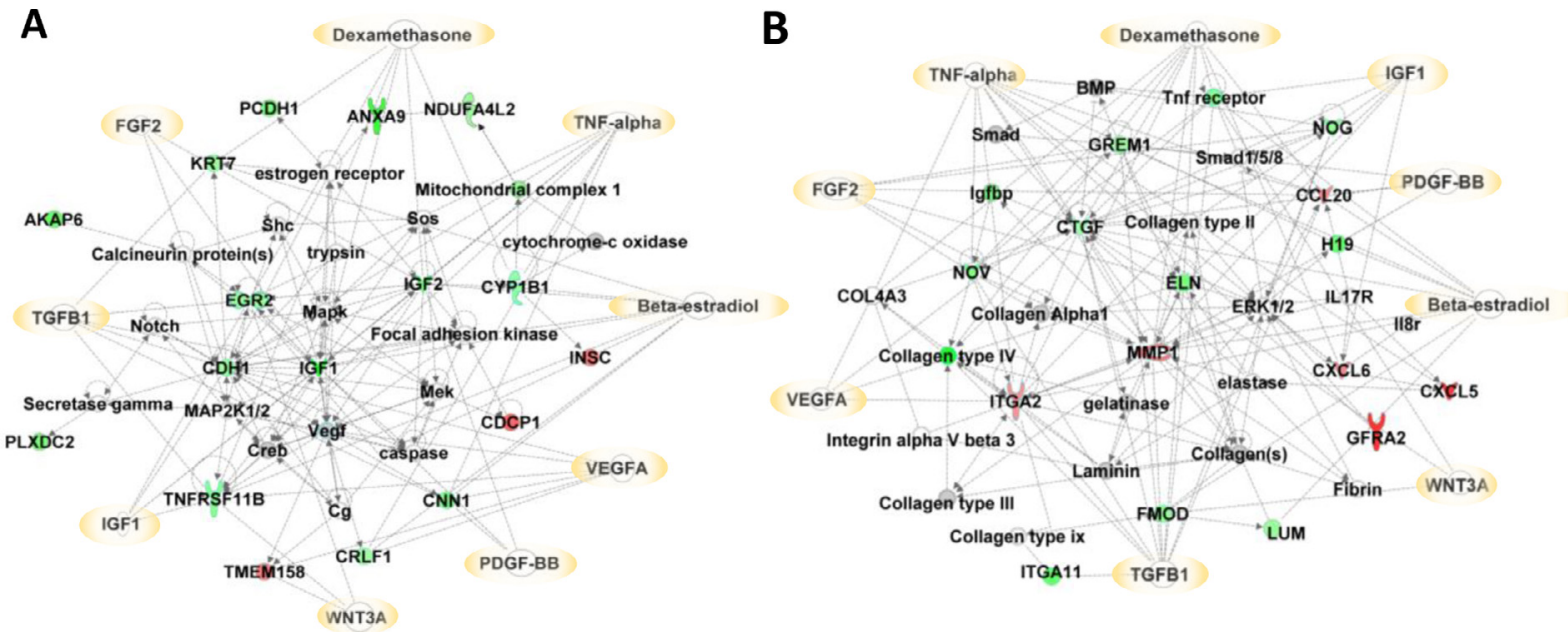

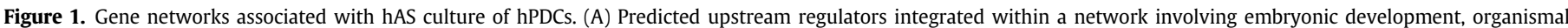

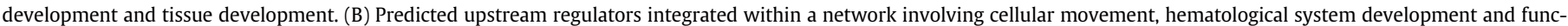

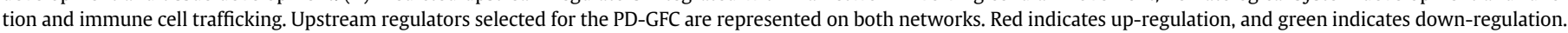

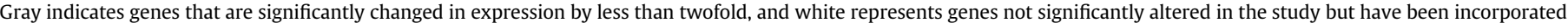
into the network through relationships with other molecules. Yellow indicates potential upstream regulators. Networks created in Ingenuity Pathway Analysis (IPA; Qiagen).

development and function, immune cell trafficking were identified with the most recorded differential genes (Figure 1).

To further assess the validity of the selected factors a literature search was carried out on each to identify any known involvement in inducing cellular proliferation, bone formation or stemness (supplementary Table IV). This resulted in nine factors being selected for further analysis, including VEGF, dexamethasone, Wnt-3a, $\beta$-estradiol, FGF2, TNF $\alpha$, TGF $\beta$, IGF-1 and PDGF-BB.

\section{Assessing the effects of the selected factors on hPDC culture dynamics}

The effect of each selected factor on hPDC biology was assessed using a "leave-one-factor-out" strategy, which was aimed at identifying the factors that induce cellular proliferation and a gene expression profile with enhanced stem and osteochondrogenic gene expression (Figure 2). hPDCs were cultured in the various conditions for 7 days. The condition containing all factors was used as a reference to evaluate the impact of each individual factor on proliferation, cellular metabolism (Figure 2B) and the expression of stem (NESTIN [29] and PRX1 [30] based on previously published data indicating their relevance in the identification of cells from mesenchymal origin with apparent plasticity), early osteogenic and chondrogenic gene markers (Figure $2 \mathrm{~A}$ ). A positive regulation upon elimination of a factor compared with the reference indicated that this factor had a negative effect on the measurement. In this regard, we identified FGF2 as a strong inducer of proliferation (2.2-fold decrease in DNA content upon its elimination, $P<0.001$ ), metabolism (1.25-fold decrease in metabolic activity) and stemness (NESTIN: 1.2-fold decrease; PRX1: 1.7-fold decrease, $P<0.05$ ); however, minimal effects were observed with regard to early osteogenic (RUNX2: 1.4-fold increase, ALP: 1.2fold increase) and chondrogenic (SOX9: 1.2-fold decrease and COL2A1: 2.2-fold increase, $P<0.001$ ) markers. TGF $\beta$, on the other hand, had a minimal effect when considering metabolism (1.1-fold increase, $P<$ $0.05)$; however, its removal decreased the expression of NESTIN (1.9fold decrease, $P<0.01$ ), RUNX2 (2.5-fold decrease, $P<0.01), A L P(6.0$ fold decrease, $P<0.001$ ) and COL2A1 (10-fold decrease, $P<0.05$ ).

TNF $\alpha$ and $\beta$-estradiol were the only molecules that when removed induced a significant increase in the committed osteoblast marker $A L P$. As the primary goal of this study was to define culture conditions that allow the expansion of hPDCs in the absence of differentiation, both were retained in the culture conditions. In the case of
IGF-1 and PDGF-BB, their removal caused minimal change in any tested parameter; however, removal caused the appearance of a heterogeneous culture and/or stress fibers within the cells and as such both were retained (supplementary Figure 2).

Eliminating dexamethasone resulted in a significant increase of all tested gene markers (NESTIN: 1.8-fold, $P<0.001$; PRX1: 1.4 -fold, $P<$ 0.001; RUNX2: 1.5-fold, $P<0.001$; SOX9: 1.86-fold, $P<0.001$; COL2A1: 0.8 -fold, $P<0.01$ ) with the exception of $A L P$ (5.0-fold decrease, $P<$ $0.001)$. With this reasoning, dexamethasone was eliminated from the growth factor cocktail. Additionally, eliminating Wnt-3a from the study significantly increased cellular proliferation (0.3-fold increase, $P<0.05$ ) with no significant effect on cellular metabolism and all tested gene markers and was thus eliminated from the study. Lastly, eliminating VEGF caused a significant increase in proliferation (0.4fold change, $P<0.01)$ and cellular metabolism (0.4-fold change, $P<$ 0.01 ), with no significant effect on tested gene markers and was thus eliminated from the study.

Using this methodology, a refined cocktail of factors was generated that included $\beta$-estradiol, FGF2, TNF $\alpha$, TGF $\beta$, IGF-1 and PDGF$\mathrm{BB}$. This was termed periosteum-derived growth factor cocktail (PDGFC). The morphology of cells cultured in hAS and PD-GFC was similar (Figure 2C) indicating potential equivalence of the culture conditions based on cell phenotype.

\section{hPDCs cultured in PD-GFC display differences in cellular morphology and lineage commitment in vitro compared with $10 \%$ FBS}

To assess the efficacy of the PD-GFC for hPDC expansion, hPDCs were treated with the PD-GFC over multiple passages to observe its effect on the cell's proliferation rate relative to $10 \%$ and $1 \%$ FBS. Culture of hPDCs in PD-GFC resulted in significantly higher cumulative population doublings over time and per passage (after passage 8 ) compared with $10 \%$ FBS and $1 \%$ FBS $(P<0.05$; Figure $3 \mathrm{~A}$, $B)$. The cells remained proliferative after passage 10 and appeared morphologically normal. Data associated with a typical passage in each of the media formulations is shown in supplementary Table V. However, it cannot be categorically stated from these data that no senescent cells were present within the cultures. With regard to cell spreading, the ratio of the cell width to length was quantified when treated with 1\% FBS, 10\% FBS and PD-GFC (Figure 3B). hPDCs cultured in PD-GFC resulted in a 3.1-fold lower 
A

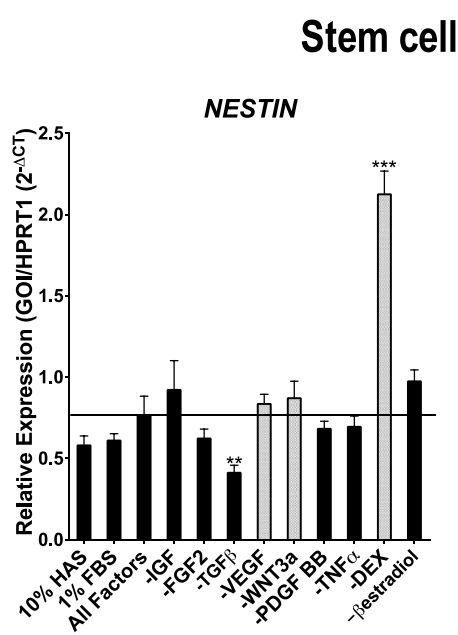

markers

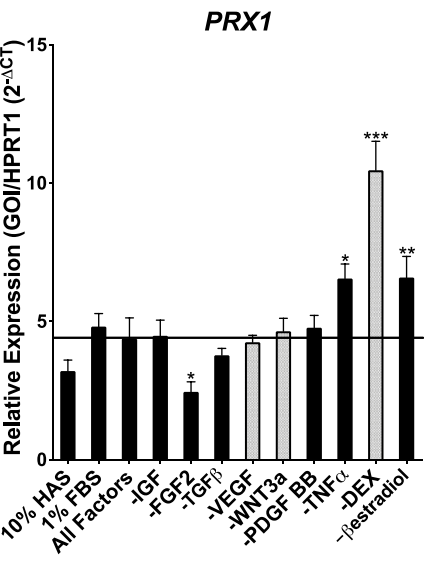

Osteogenic markers
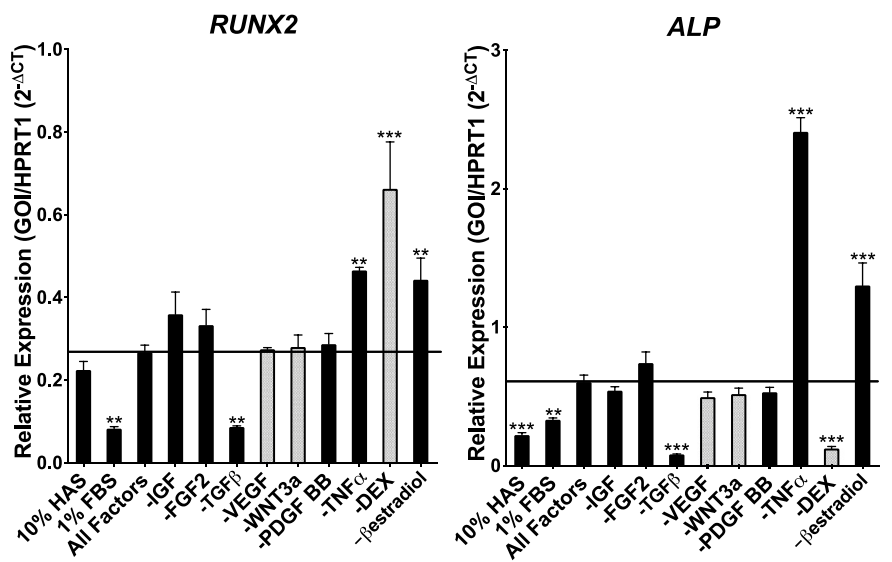

Chondrogenic markers

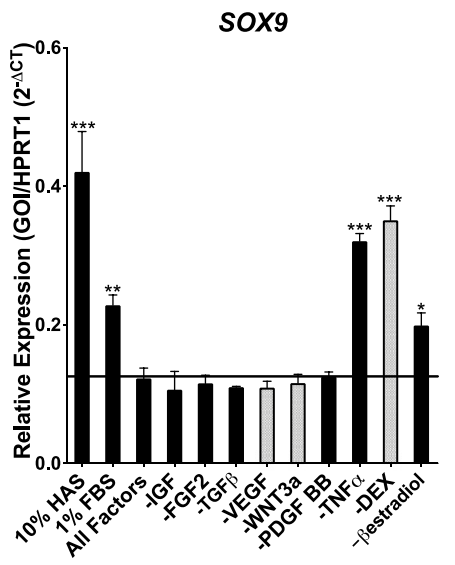

COL2A1
B
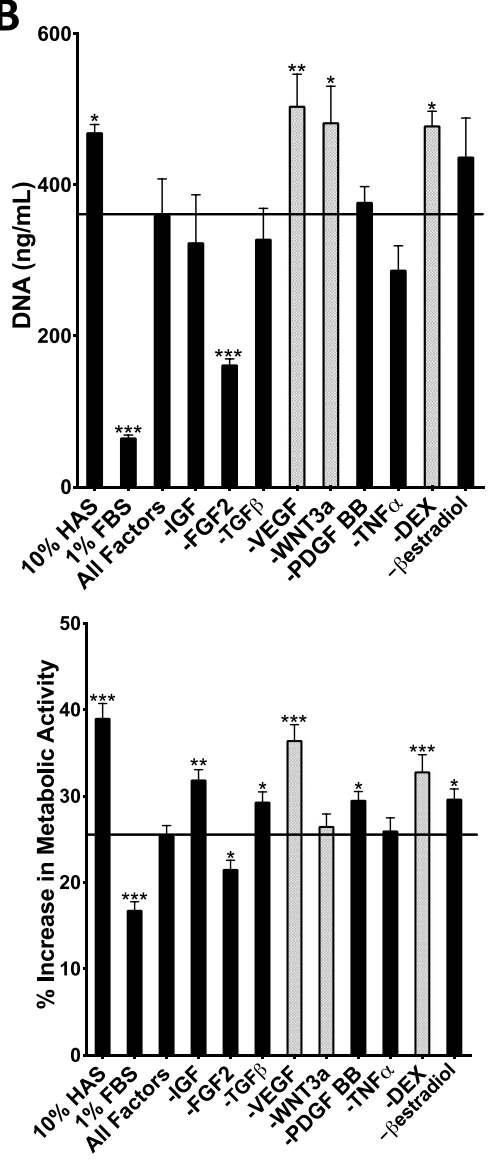

C
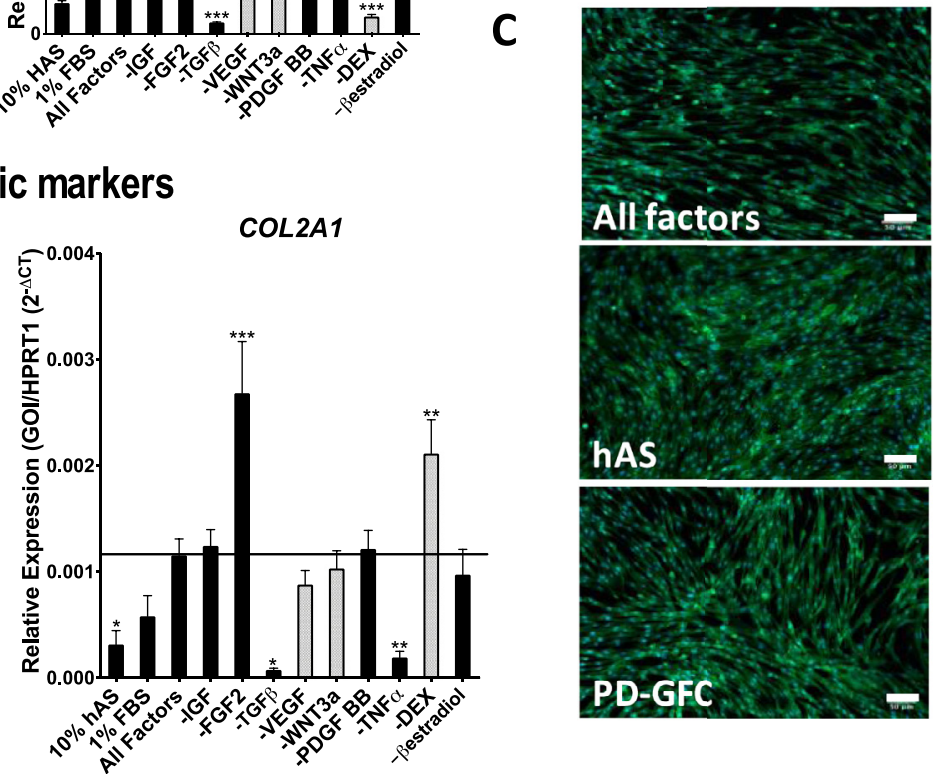
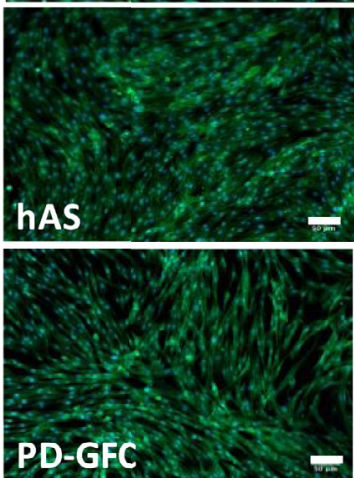

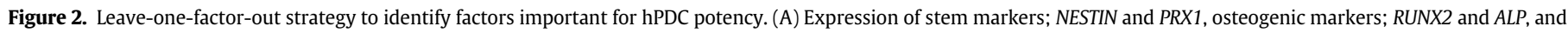

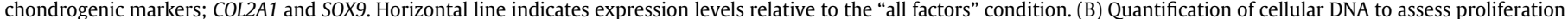

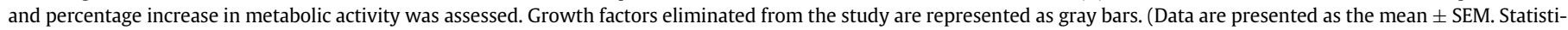

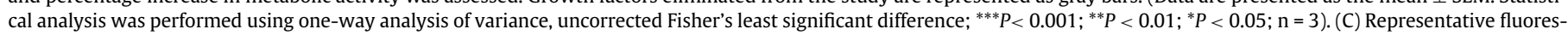

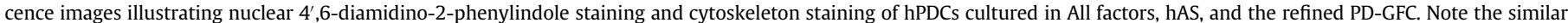
morphology of hPDCs cultured in PD-GFC and hAS (scale bar $=50 \mu \mathrm{m}$ ).

ratio than when cultured in $10 \%$ FBS $(P<0.001$; Figure $3 C)$, which follows the same trend as previously published for hAS-cultured cells [11]. Furthermore, expression of the mesenchymal stromal marker NESTIN was higher at each passage when cultured in PD-GFC compared to $10 \%$ FBS $(1.9, P<0.01 ; 3.3, \mathrm{P}<0.001 ; 2.0$,
$\mathrm{P}<0.01$; and 2.6-fold, $\mathrm{P}<0.001$ higher at passage $7-10$ respectively). In cells treated with the PD-GFC relative to $10 \%$ FBS, PRX1 displayed an increase in expression in PD-GFC treated cells at passage 7 (1.3-fold, nonsignificant [ns]), 8 (1.3-fold, $P<0.05$ ), 9 (1.2-fold, ns) and $10(2.0, P<0.001)$ compared with $10 \%$ FBS 

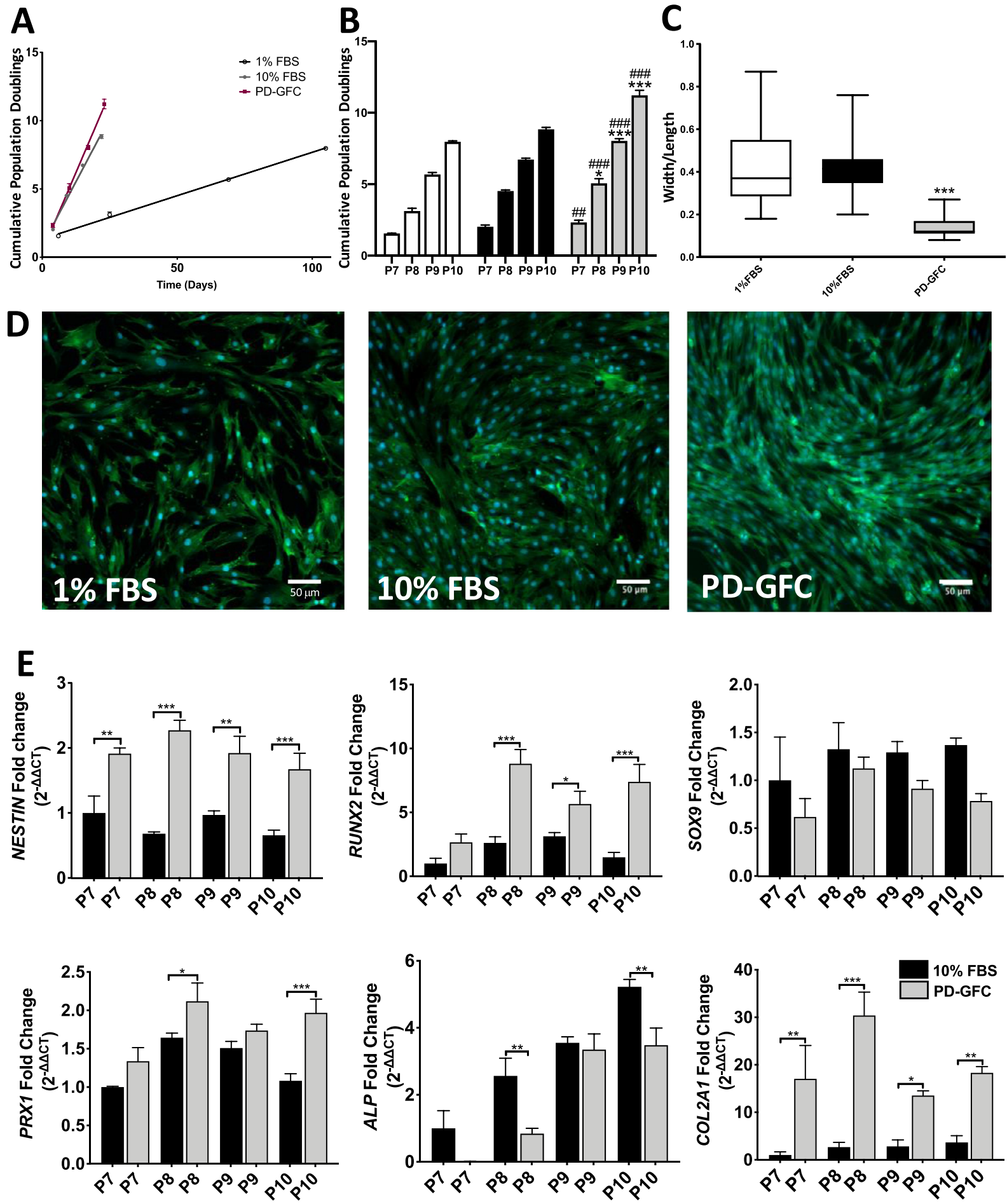

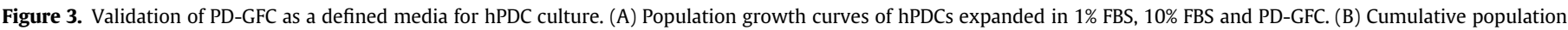

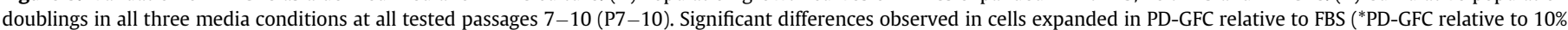

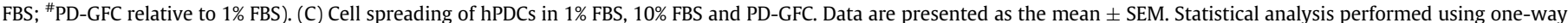

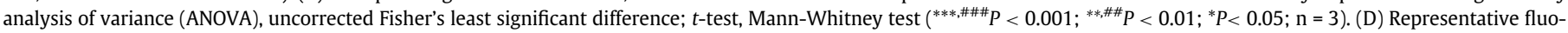

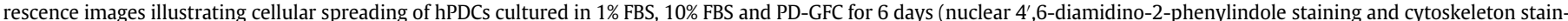

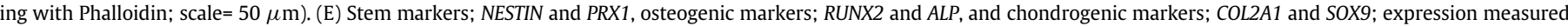

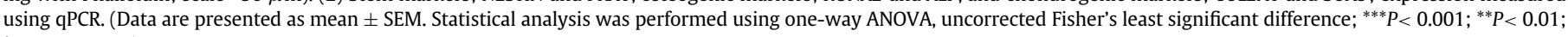
${ }^{*} P<0.05 ; \mathrm{n}=3$ )

(Figure 3D). The expression of the osteogenic transcription factor RUNX2 was increased in the PD-GFC treated cells, with a significant increase observed at passages 8 (3.4-fold, $P<0.001$ ), 9 (1.8fold, $P<0.05)$ and 10 (5.0-fold, $P<0.001)$. Interestingly, $A L P$ expression was lower in PD-GFC treated cells and significantly lower at passage 8 (3.0-fold, $P<0.01$ ) and 10 (1.5-fold, $P<0.01$ ). With regard to chondrogenic markers, SOX9 displayed a lower expression profile at passages 7 to 10 in cells treated with PDGFC, although not significant. COL2A1 expression was, however, significantly up-regulated in PD-GFC treated cells at passage 7 

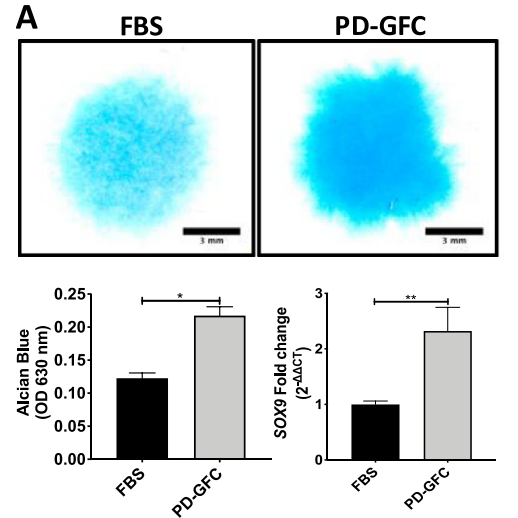
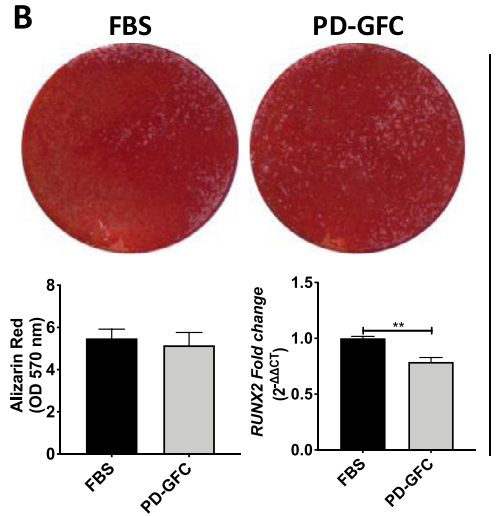

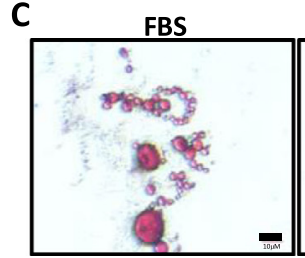

PD-GFC
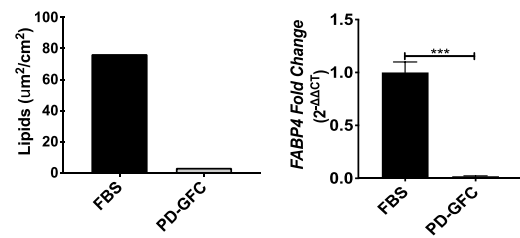

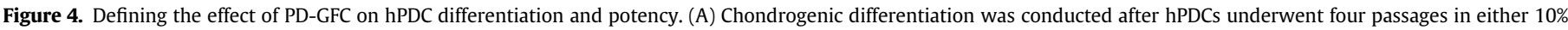

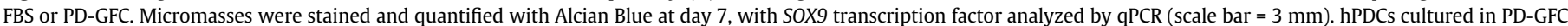

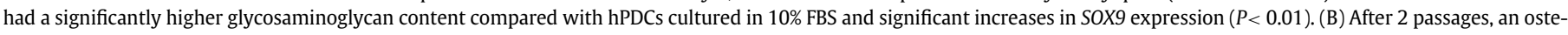

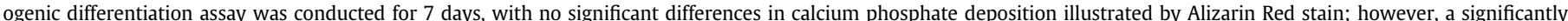

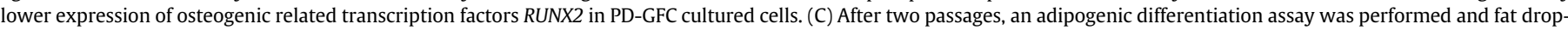

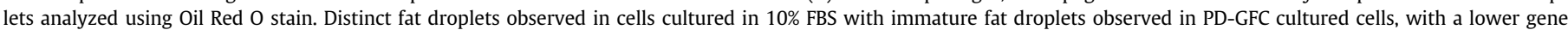

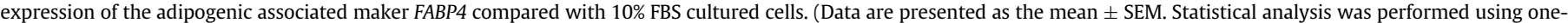
way analysis of variance, uncorrected Fisher's least significant difference; ${ }^{* * *} \mathrm{P}<0.001 ;{ }^{* *} \mathrm{P}<0.01 ;{ }^{*} \mathrm{P}<0.05 ; \mathrm{n}=3, \mathrm{scale}$ bar $\left.=10 \mu \mathrm{m}\right)$.

through 10 (17.0 $(P<0.01), 11.4(P<0.001), 4.8(P<0.05), 5.0(P<$ 0.01 )-fold, respectively) relative to $10 \%$ FBS.

\section{Expansion in PD-GFC maintains hPDC osteochondrogenic potential}

After expansion of hPDCs through multiple passages (as mentioned earlier), a subset of cells was subjected to standard chondrogenic, osteogenic and adipogenic differentiation conditions. The data illustrated in Figure $4 \mathrm{~A}$ is a representation of the chondrogenic differentiation assay. The micromass cultures treated with standard chondrogenic factors resulted in a 1.8 -fold increase $(P<0.05)$ in Alcian Blue stain, indicative of proteoglycan deposition in cells previously expanded in the PD-GFC relative to 10\% FBS (Figure 4A). The early chondrogenic transcription factor SOX9 was significantly up-regulated (2.3-fold, $P<0.001)$ in the PD-GFC primed cells compared with $10 \%$ FBS cultured cells.

Osteogenic differentiation was conducted for 7 days (Figure 4B), with no significant differences in calcium phosphate deposition illustrated by an Alizarin Red stain between the two expansion medias. A modest reduction in the expression of the osteogenic transcription factor RUNX2 (1.3-fold, $P<0.01)$ was observed in PD-GFC cultured cells compared with $10 \%$ FBS.

The cells were further subjected to an adipogenic differentiation assay for 21 days (Figure 4C). An Oil Red $\mathrm{O}$ stain was performed to identify the formation of fat droplets. Cells cultured in $10 \%$ FBS before the differentiation assay resulted in the formation of large fat droplets. However, cells cultured in PD-GFC resulted in immature fat droplet formation after the 21-day period, further confirmed by a 23.4-fold decrease in lipid droplets (Figure 4C). A 52.6-fold $(P<0.001)$ decrease in the adipogenic marker FABP4 was observed in PD-GFC expanded cells relative to $10 \%$ FBS.

\section{hPDCs encapsulated within a 3D collagen type 1 matrix and cultured in $P D-G F C$ display periosteum-like characteristics}

hPDCs were embedded within a 3D collagen type 1 matrix. The aim of this study was to further recapitulate the in vivo periosteum microenvironment by incorporating the cell populations in optimized culture conditions in a relevant matrix. One hundred thousand cells were either seeded in a 2D monolayer or embedded within $10 \%$ (plastic compressed) 3D collagen type 1 matrix. After a 14-day culture, the constructs were paraffin embedded, sectioned and stained with hematoxylin and eosin to observe the collagen and cell distribution. Upon plastic compression of the collagen gels, a dense layer of collagen was visible on the fluid leaving surface (FLS) compared with the rest of scaffold (Figure 5B). The $10 \%$ FBS cultured constructs had cells dispersed throughout the matrix. However, when cultured in PD-GFC, more hPDCs were visible within the construct with the majority of cells aligned along the FLS of the scaffold. Interestingly, this did not occur when using a lower cell density of 30000 cells (supplementary Figure 3A). Intriguingly, the organization of the cells mirrored that of native periosteum (Figure 5A). Furthermore, when comparing the gene expression profile of this biomimetic construct to cells cultured in standard conditions, an up-regulation of genes associated with periosteal biology was observed with the exception of PRX1 (supplementary Figure 3B). We hypothesized that this cell organization was due to the biophysical properties of this region, and as such we set out to analyze the morphology of the collagen fibrils and the matrix stiffness of both the native periosteum and hPDC laden collagen type 1 scaffold following 14 days culture in PD-GFC. As seen in Figure 5E, AFM stiffness measurements of either side of the scaffold revealed no difference, unlike native periosteum where the fibrous layer was 1.47 -fold stiffer than the cambium layer. Interestingly, the scaffold stiffness was in the same range as that observed with periosteum (scaffold $=5.40-5.45$ $\mathrm{GPa}$, periosteum $=4.55-6.69 \mathrm{GPa}$ ). With regard to collagen fibril morphology, collagen fibril structure and orientation was similar between the FLS and cambium layer, respectively. A similar fibril morphology was also observed between the bottom of the scaffold and the fibrous layer of the periosteum (Figure 5C).

\section{Identification of signal transduction factors associated with PD-GFC cultured hPDCs}

To further determine the underlying mechanisms involved in PDGFC culturing of hPDCs in a 3D environment, cells were embedded in a 3D collagen type 1 matrix and cultured in either 10\% FBS or PD-GFC for 14 days. RNA was extracted and changes in gene expression was observed using the human stem cell signaling array. Of the 84 genes examined, 9 were significantly up-regulated in PD-GFC cultured cells relative to FBS, and 17 were significantly down-regulated in PD-GFC cultured cells relative to FBS with a twofold or more change in expression, illustrated in the volcano plot (Figure 5F). The data were further interrogated using IPA to define the likely gene network associated with the transcriptional profile. The gene network shown in Figure 5G represents genes from the PCR array that were either up- (red) or down-regulated (green), with additional genes that are likely to play a role also represented. It was noted that TGF superfamily (ACVR2B, ACVR1B, SMAD1 and 


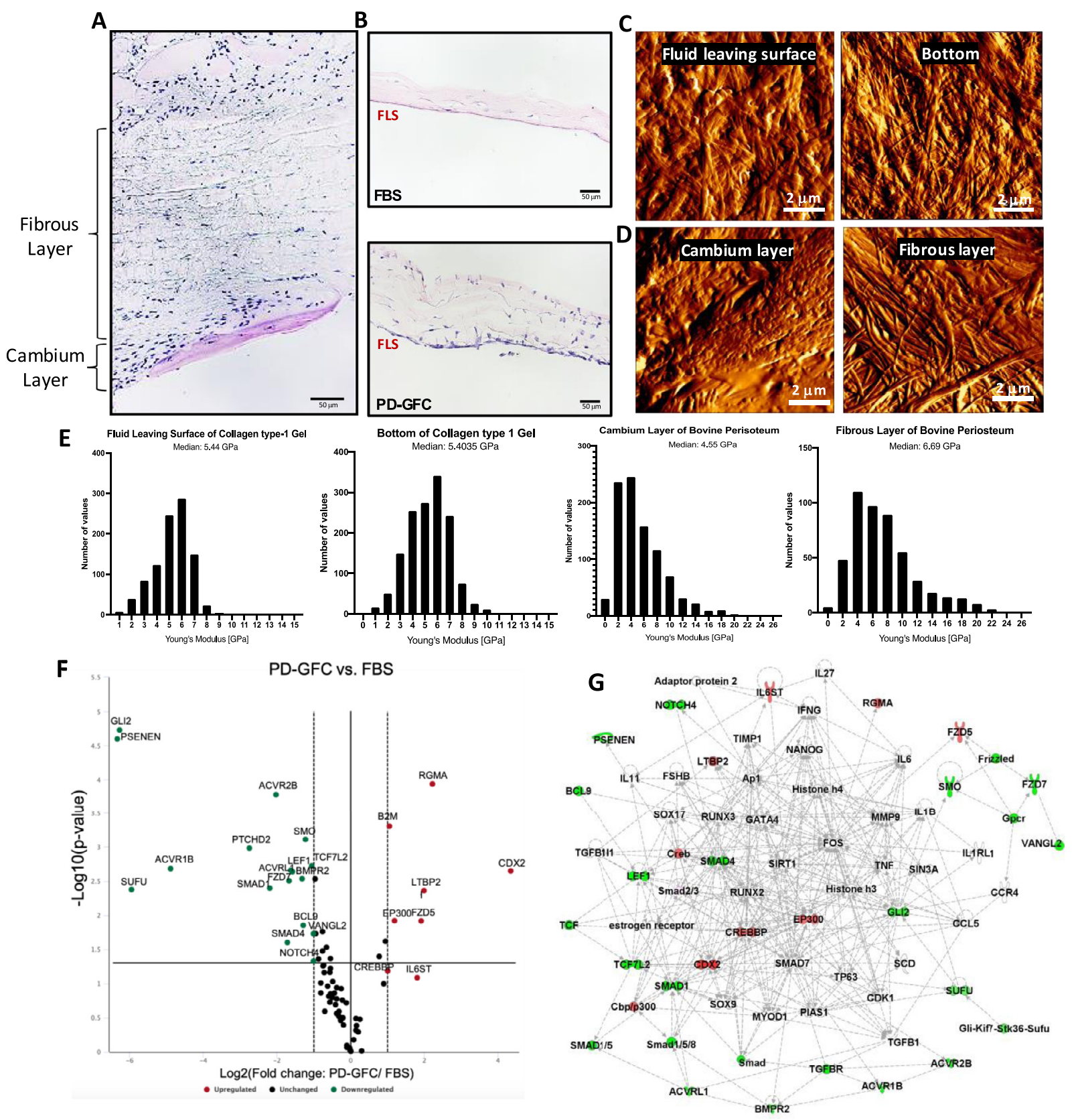

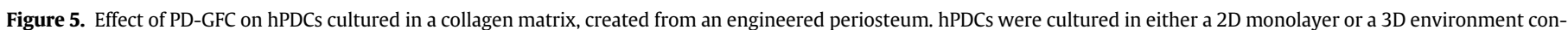

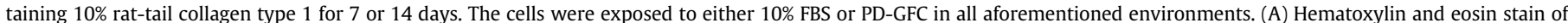

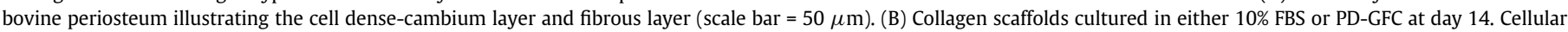

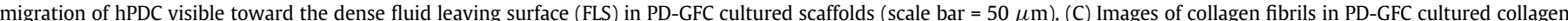

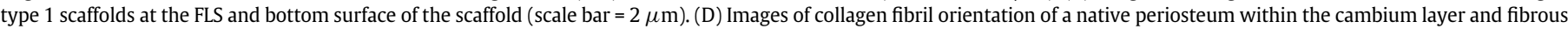

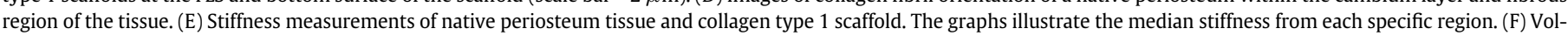

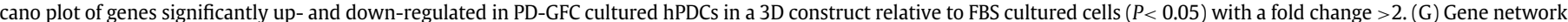
representing various signaling interactions between genes that were significantly up- and down-regulated in FBS versus PD-FC cultured constructs (created in IPA).

SMAD4), WNT signaling (FZD7, TCF and LEF1) and Hedgehog (GLI2, PTCHD2) were down-regulated with an up-regulation of CREB-associated pathways (CREBBP and EP300).

\section{Discussion}

The identification of gene networks that are activated during stem cell expansion while retaining potency is an essential step in tissue engineering and regenerative medicine. Although generic defined culture conditions have been commercialized, many of these contain a proprietary formulation of growth factors and small molecules, and as such delineating data generated using these media remains difficult. We have previously demonstrated the superiority of hAS over FBS in supporting hPDC expansion, osteochondral differentiation and in vivo bone tissue formation [11]. However, the specific signals involved in this effect are largely unknown. Through the comparison of gene expression in hPDCs cultured in hAS and FBS, we have shown that the in vitro molecular signature associated with hAS-induced identity and potency can be used to develop defined conditions for hPDC culture. Furthermore, these culture conditions can be combined with high-density collagen 3D matrices to promote periosteum-like tissue formation in vitro. Indeed, mimicking the periosteum 
microenvironment as a means of facilitating bone regeneration in vivo is a credible method of introducing cells into bone defects that lack the key components to initiate the repair process.

Serum contains more than 1000 components, including proteins, lipids, carbohydrates, growth factors, enzymes and other constituents that are still undefined. The precise effect of serum is cell-type specific; however, it generally regulates cell growth and phenotype. As previously stated, we have shown that serum from human sources outperforms those from bovine sources when culturing hPDCs, however the mechanism of this effect and factors that mediate it are largely unexplored. Indeed, although EGF, FGF-2, PDGF, VEGF and IGF-1 have been identified as key growth factors in human serum [31], whether all or only some confer hPDC identity and potency is unknown. Interestingly, four of these factors are represented in the nine factors identified from transcriptomic analysis of hAS-cultured hPDCs (PDGF-BB, $\beta$-estradiol, Wnt-3a, IGF-1, TNF $\alpha$, TGF $\beta$, VEGF, FGF2 and dexamethasone). Furthermore, each of these nine factors has also been implemented in stem cell culture and increased potency. There is already a wealth of literature suggesting these factors are important in (i) delaying MSC senescence (estrogen and dexamethasone) [32,33]; (ii) improving proliferation (estrogen, dexamethasone, PDGF, Wnt-3a, VEGF and FGF) [34-38]; and (iii) regulating osteo/chondro commitment (TNF, TGF $\beta$ and IGF) [37,39-41]. Involvement of each of the selected factors in stem cell/bone biology is summarized in supplementary Table IV. Of course, the validity of each of these factors for increasing hPDC potency, and potential interactions, requires careful testing empirically.

In an attempt to create a cocktail of growth factors that stimulate robust expansion of hPDCs with improved potency, a take-one-away methodology was used. It was predicted that when analyzed with respect to cell proliferation and gene expression profile, a conclusion on the specific factor's importance could be made. For example, the inclusion of Wnt-3a in the initial growth factor list was predominantly a result of its significance as an upstream regulator in the transcriptomic analysis and previous studies indicating its role in bone metabolism and MSC proliferation. However, studies have reported contradictory results with regard to Wnt-3a and osteocommitment, which appears to be dependent of cell type and stage of differentiation. Indeed, when considering adipose derived MSCs, Wnt-3a increased the early osteoblast marker $A L P$, but mature osteoblast markers were not increased [42]. Conversely, a positive effect on multipotent characteristics and proliferative state has been suggested for bone marrow MSCs when FGF2 and Wnt-3a are combined [43]. Nevertheless, herein, eliminating Wnt-3a had a positive effect on cell proliferation but no effect on any stem or osteochondrogenic markers.

The elimination of dexamethasone resulted in a significant up-regulation of all tested gene markers (with the exception of $A L P$ ) as well as enhancing cellular proliferation and metabolism. Due to its dosedependent mechanism, dexamethasone has previously been reported to both promote and arrest osteogenic differentiation. Interestingly herein, dexamethasone appeared to have a negative effect on hPDC biology. Indeed, we have previously reported that dexamethasone overall has a negative effect on osteogenic differentiation, unless in combination with 10\% FBS [44]. Likewise, eliminating VEGF from culture resulted in minimal alteration in analyzed markers but did promote proliferation and metabolism relative to all factors. VEGF is known to promote osteogenic differentiation; however, a lack of effect when considering PDCs may represent a cell-specific mechanistic difference. In summary, eliminating a combination of Wnt-3a, dexamethasone and VEGF had minimal negative effect on the cell's stem, osteogenic and chondrogenic markers relative to all other factors, with the cell's morphology resembling that of cells cultured in hAS. This allowed the formulation of the defined PD-GFC culture conditions.

A significant difference was observed in the proliferation of cells cultured in PD-GFC relative to FBS, as previously reported with hAS
[11]. In addition, cell morphology and spreading of hPDCs cultured in PD-GFC mimicked that of cells cultured in hAS [11]. Furthermore, hPDCs cultured in PD-GFC demonstrated significant increases in stem cell markers PRX1 and NESTIN over serial passages and an increase in the chondrocyte marker COL2A1 and osteoblast marker RUNX2. When expanded in PD-GFC, the resultant cells had a greater propensity to deposit GAGs when differentiated toward chondrocytes, and a decrease in fat droplet content when differentiated toward adipocytes, compared with cells cultured in $10 \%$ FBS. Indeed, this mirrored the results with hAS, with the exception of CaP deposition where an increase was observed compared with FBS [45]. However, hPDC optimized osteogenic differentiation conditions were used herein, which were not used in the earlier study. Interestingly, periostin (POSTN) has been proposed as a key regulator of the cell's differentiation capacity [46]. Indeed, culturing hPDCs in the PD-GFC caused a significant up-regulation of POSTN (not shown) compared with FBS-cultured cells, which may account in part for the enhanced differentiation capacity of the cells.

In an attempt to further mimic the periosteum in vitro, the cells were incorporated within a collagen type 1 matrix. Interestingly, we observed a distinct migration of cells to the fluid leaving surface of the collagen scaffold by day 14 (illustrated on the bottom edge of the collagen scaffolds in Figure 5B) when culturing the cells in PD-GFC. This resembled the cell distribution seen in native periosteum where cell density is increased in the cambium layer of the periosteum (as illustrated in Figure 5A). A significant increase in expression $\left({ }^{* * *} P<0.001\right)$ of the periosteum associated gene cathepsin K (CTSK; data not shown) was seen in scaffolds cultured in PD-GFC relative to FBS by day 14 CTSK is a collagenase enzyme that has recently been shown to be expressed in a subpopulation of periosteal stem cells [6]; however, in this instance, it may be aiding matrix remodeling, thus allowing cell movement. In an attempt to define the cues relating to cell movement, we assessed whether matrix stiffness was regulating migration. Interestingly, no difference was observed between the stiffness of the two surfaces of the scaffold, although they were in the same stiffness range as native periosteum. However, upon analysis of collagen orientation of both the scaffold and periosteum, a similar interwoven morphology was observed between the cambium layer and FLS of the scaffold. Additionally, a similar longitudinally oriented collagen fibre morphology was observed between the fibrous layer and bottom of the scaffold. As such, the matrix ultrastructure could be a potential cue in regulating the migration of cells to the various regions of the scaffold. Indeed, contact guidance and bidirectional migration along aligned collagen fibers is a known phenomenon in both wound-healing and cancer biology [47]. The increased population of hPDCs at the FLS when cultured in the PD-GFC suggests a synergistic effect of the dense interwoven collagen matrix as well as the presence of active cytokines and growth factors that may have encouraged the accumulation of PDCs. This pattern and localization of cells in a collagen matrix is reminiscent of the cambium layer of the periosteum.

When assessing the signaling transduction pathways associated with the aforementioned culture system, down-regulated pathways were predominantly associated with TGF- $\beta$ and WNT, and up-regulated pathways associated with CREB. Interestingly, up-regulated genes include EP300 and CREBBP. EP300 is a histone acetyltransferase and coactivator and plays a vital role in physiological processes including the regulation of embryonic stem cell self-renewal and pluripotency [48]. CREBBP and EP300 are recruited by Nanog (an identified hub gene) through a physical interaction to the Nanog binding loci and play a pivotal role in maintaining a proliferative and undifferentiated population of stromal stem cells [49]. Additionally, CREB-mediated signaling has been implicated in the expansion and self-renewal of muscle stem cells to preserve stem cell function [50]. Interestingly, CREB has also been linked with in vivo bone formation, with prolonged activation of the cAMP pathway by either dibutryl-cAMP [51] or forskolin [52] shown to stimulate in vitro and in vivo bone formation from human MSCs. 
Furthermore, CREB is associated with periosteal biology. Indeed, PDGF secreted by TRAP $^{+}$mononuclear cells have been shown to maintain the periosteum microenvironment potentially though its induction of pCREB binding to the periostin promoter [53].

As previously mentioned, an overall decrease in WNT signaling was apparent in cells cultured in the PD-GFC relative to FBS. With reference to the initial take-one-away study, the presence of Wnt-3a had a negative effect on cell proliferation and as such was eliminated from further analysis. Interestingly, the six growth factors selected in addition to the 3D matrix induced an autonomous down-regulation of WNT signalling, further highlighting the need to eliminate this pathway. Indeed, it is known that activation of WNT signaling leads to periosteal bone formation, presumably as a direct result of periosteal stem cell differentiation [54]. TGF- $\beta$ signalling was also downregulated in this system. Specifically, the type 1 receptor $A C V R 1 B$ and the type 2 receptor $A C V R 2 B$ were down-regulated. These two receptors form the signaling complex for activins including activin a, activin $b$ and nodal. This signaling pathway functions through the interaction of Smad2/3 with Smad4, which is also down-regulated in our analysis. Interestingly, activin/nodal signaling has been implicated in both pluripotent and adult stem cell fate choices [55].

Overall, the PD-GFC developed in the current study represents a defined media for the expansion of hPDCs in vitro, while retaining cell identity and improving potency. Incorporating PD-GFC cultured cells in a collagen type 1 matrix mimicked native periosteum through cell localization. Regulation of specific pathways in response to PDGFC and the environment suggest the enrichment or modification of hPDCs to mimic the periosteal progenitor niche. These data could provide the basis for an "off-the-shelf" periosteum mimetic to replace periosteal grafting in vivo; however, efficacy of such a construct would first need to be tested preclinically.

\section{Declaration of Competing Interest}

The authors have no commercial, proprietary, or financial interest in the products or companies described in this article.

\section{Author Contributions}

Conception and design of the study: RA, UC, HR, FL, SR. Acquisition of data: RA, MS, SR. Analysis and interpretation of data: RA, MS, UC, HR, FL, SR. Drafting or revising the manuscript: RA, MS, UC, HR, FL, SR. All authors have approved the final article.

\section{Acknowledgments}

The authors acknowledge Dr. Laurent Bozec and Mr. Adam Strange (UCL, United Kingdom) for their assistance with the AFM experiments detailed within this article and the Nucleomics Core (KU Leuven) for conducting/analyzing the RNAseq.

\section{Supplementary materials}

Supplementary material associated with this article can be found in the online version at doi:10.1016/j.jcyt.2020.03.434.

\section{References}

[1] Lin Z, Fateh A, Salem DM, Intini G. Periosteum: biology and applications in craniofacial bone regeneration. J Dent Res 2014;93:109-16.

[2] Ito Y, Fitzsimmons JS, Sanyal A, Mello MA, Mukherjee N, O’Driscoll SW. Localization of chondrocyte precursors in periosteum. Osteoarthr Cartil 2001:9:215-23.

[3] Dwek JR. The periosteum: what is it, where is it, and what mimics it in its absence? Skeletal Radiol 2010;39(4):319.

[4] Evans SF, Chang H, Knothe Tate ML. Elucidating multiscale periosteal mechanobiology: a key to unlocking the smart properties and regenerative capacity of the periosteum? Tissue Eng Part B Rev 2013;19(2):147-59.
[5] Knothe UR, Dolejs S, Miller MR, Knothe Tate ML. Effects of mechanical loading patterns, bone graft, and proximity to periosteum on bone defect healing. J Biomech 2010;43(14):2728-37.

[6] Debnath S, Yallowitz AR, McCormick J, Lalani S, Zhang T, Xu R, et al. Discovery of a periosteal stem cell mediating intramembranous bone formation. Nature 2018:562:133-9.

[7] Roberts SJ, van Gastel N, Carmeliet G, Luyten FP. Uncovering the periosteum for skeletal regeneration: the stem cell that lies beneath. Bone 2015;70:10-8.

[8] Petrochenko P, Narayan RJ. Novel approaches to bone grafting: porosity, bone morphogenetic proteins, stem cells, and the periosteum. J Long Term Eff Med Implants 2010;20(4):303-15

[9] Geris L, Reed AAC, Vander Sloten J, Simpson AHRW, Van Oosterwyck H. Occurrence and treatment of bone atrophic non-unions investigated by an integrative approach. PLoS Comput Biol 2010;6:e1000915.

[10] Knothe UR, Springfield DS. A novel surgical procedure for bridging of massive bone defects. World J Surg Oncol 2005;3(1):7.

[11] Roberts SJ, Owen HC, Tam WL, Solie L, Van Cromphaut SJ, Van den Berghe G, et al. Humanized culture of periosteal progenitors in allogeneic serum enhances osteogenic differentiation and in vivo bone formation. Stem Cells Transl Med 2014:3:218-28.

[12] Colnot C. Skeletal cell fate decisions within periosteum and bone marrow during bone regeneration. J Bone Miner Res 2009;24:274-82.

[13] Roberts SJ, Geris L, Kerckhofs G, Desmet E, Schrooten J, Luyten FP. The combined bone forming capacity of human periosteal derived cells and calcium phosphates. Biomaterials 2011;32:4393-405

[14] De Bari C, Dell'Accio F, Vanlauwe J, Eyckmans J, Khan IM, Archer CW, et al. Mesenchymal multipotency of adult human periosteal cells demonstrated by single-cell lineage analysis. Arthritis Rheum 2006;54:1209-21.

[15] Cimino M, Gonçalves RM, Barrias CC, Martins MCL. Xeno-free strategies for safe human mesenchymal stem/stromal cell expansion: supplements and coatings. Stem Cells Int 2017:2017:6597815.

[16] Bjare U. Serum-free cell culture. Pharmacol Ther 1992;53(3):355-74.

[17] Zheng X, Baker H, Hancock WS, Fawaz F, McCaman M, Pungor E. Proteomic analysis for the assessment of different lots of fetal bovine serum as a raw material for cell culture. Part IV. Application of proteomics to the manufacture of biological drugs. Biotechnol Prog. 2006;22(5):1294-300.

[18] Simonetti AB, Englert GE, Campos K, Mergener M, de David C, de Oliveira AP, et al. Nanobacteria-like particles: a threat to cell cultures. Brazilian J Microbiol 2007 ;38 (1):153-8. [cited 2019 Mar 18].

[19] Van Der Valk J, Mellor D, Brands R, Fischer R, Gruber F, Gstraunthaler G, et al. The humane collection of fetal bovine serum and possibilities for serum-free cell and tissue culture. Toxicol In Vitro 2004;18(1):1-12.

[20] Brunner D, Frank J, Appl H, Schöffl H, Pfaller W, Gstraunthaler G. Serum-free cell culture: the serum-free media interactive online database. ALTEX 2010;27(1):53-62.

[21] Gupta P, Hall GN, Geris L, Luyten FP, Papantoniou I. Human platelet lysate improves bone forming potential of human progenitor cells expanded in microcarrier-based dynamic culture. Stem Cells Transl Med 2019;8(8):810-21.

[22] Van der Valk J, Bieback K, Buta C, Cochrane B, Dirks WG, Fu J, et al. Fetal bovine serum (FBS): past-present-future. ALTEX 2018;35(1):99-118.

[23] Chen Y, Sonnaert M, Roberts SJ, Luyten FP, Schrooten J. Validation of a PicoGreenbased DNA quantification integrated in an RNA extraction method for twodimensional and three-dimensional cell cultures. Tissue Eng Part C Methods 2012;18:444-52.

[24] Livak KJ, Schmittgen TD. Analysis of relative gene expression data using realtime quantitative PCR and the 2(-Delta Delta $C(T)$ ) Method. Methods $2001 ; 25(4): 402-8$.

[25] Magdeldin T, López-Dávila V, Pape J, Cameron GWW, Emberton M, Loizidou M, et al. Engineering a vascularised 3D in vitro model of cancer progression. Sci Rep 2017;7:44045.

[26] Hadjipanayi E, Ananta M, Binkowski M, Streeter I, Lu Z, Cui ZF, et al. Mechanisms of structure generation during plastic compression of nanofibrillar collagen hydrogel scaffolds: towards engineering of collagen. J Tissue Eng Regen Med 2011;5(7):505-19.

[27] Brown RA, Wiseman M, Chuo CB, Cheema U, Nazhat SN. Ultrarapid engineering of biomimetic materials and tissues: fabrication of nano- and microstructures by plastic compression. Adv Funct Mater 2005;15:1762-70.

[28] Rio DC, Ares M, Hannon GJ, Nilsen TW. Purification of RNA using TRIzol (TRI Reagent). Cold Spring Harb Protoc 2010;2010(6).

[29] Méndez-Ferrer S, Michurina TV, Ferraro F, Mazloom AR, MacArthur BD, Lira SA et al. Mesenchymal and haematopoietic stem cells form a unique bone marrow niche. Nature 2010;466:829-34.

[30] Ouyang Z, Chen Z, Ishikawa M, Yue X, Kawanami A, Leahy P, et al. Prx1 and 3.2kb Col1a1 promoters target distinct bone cell populations in transgenic mice. Bone 2014;58:136-45

[31] Montali M, Barachini S, Panvini FM, Carnicelli V, Fulceri F, Petrini I, et al, Growth factor content in human sera affects the isolation of mesangiogenic progenitor cells (MPCs) from human bone marrow. Front Cell Dev Biol 2016;4:114.

[32] Hong L, Zhang G, Sultana H, Yu Y, Wei Z. The effects of 17- $\beta$ estradiol on enhancing proliferation of human bone marrow mesenchymal stromal cells in vitro. Stem Cells Dev 2011;20:925-31.

[33] Yun SP, Lee MY, Ryu JM, Song CH, Han HJ. Role of HIF-1alpha and VEGF in human mesenchymal stem cell proliferation by 17beta-estradiol: involvement of PKC, PI3K/Akt, and MAPKs. Am J Physiol Cell Physiol 2009;296:C317-26. 
[34] Xiao Y, Peperzak V, van Rijn L, Borst J, de Bruijn J. Dexamethasone treatment during the expansion phase maintains stemness of bone marrow mesenchymal stem cells. J Tissue Eng Regen Med 2010;4(5):374-86.

[35] De Boer J, Wang HJ, Van Blitterswijk C. Effects of Wnt signaling on proliferation and differentiation of human mesenchymal stem cells. Tissue Eng 2004;10:393-401.

[36] Lienemann PS, Devaud YR, Reuten R, Simona BR, Karlsson M, Weber W, et al. Locally controlling mesenchymal stem cell morphogenesis by 3D PDGF-BB gradients towards the establishment of an in vitro perivascular niche. Integr Biol 2015;7:101-11.

[37] Huang Z, Ren P-G, Ma T, Smith RL, Goodman SB. Modulating osteogenesis of mesenchymal stem cells by modifying growth factor availability. Cytokine 2010;51:305-10.

[38] Ahn H-J, Lee W-J, Kwack K, Kwon YD. FGF2 stimulates the proliferation of human mesenchymal stem cells through the transient activation of JNK signaling. FEBS Lett 2009 ;583(17):2922-6.

[39] Croes M, Oner FC, Kruyt MC, Blokhuis TJ, Bastian O, Dhert WJA, et al. Proinflammatory mediators enhance the osteogenesis of human mesenchymal stem cells after lineage commitment. PLoS One 2015;10:e0132781.

[40] Ng F, Boucher S, Koh S, Sastry KSR, Chase L, Lakshmipathy U, et al. PDGF, TGF-beta, and FGF signaling is important for differentiation and growth of mesenchymal stem cells (MSCs): transcriptional profiling can identify markers and signaling pathways important in differentiation of MSCs into adipogenic, chondrogenic, and o. Blood 2008;112:295-307.

[41] Minuto F, Palermo C, Arvigo M, Barreca AM. The IGF system and bone. J Endocrinol Invest 2005;28:8-10.

[42] Batsali AK, Pontikoglou C, Koutroulakis D, Pavlaki KI, Damianaki A, Mavroudi I, et al Differential expression of cell cycle and WNT pathway-related genes accounts for differences in the growth and differentiation potential of Wharton's jelly and bone marrow-derived mesenchymal stem cells. Stem Cell Res Ther 2017;8:102.

[43] Narcisi R, Cleary MA, Brama PAJ, Hoogduijn MJ, Tüysüz N, Ten Berge D, et al. Longterm expansion, enhanced chondrogenic potential, and suppression of endochondral ossification of adult human MSCs via WNT signaling modulation. Stem Cell Rep 2015;4(3):459-72.
[44] Roberts SJ, Chen Y, Moesen M, Schrooten J, Luyten FP. Enhancement of osteogenic gene expression for the differentiation of human periosteal derived cells. Stem Cell Res 2011:7:137-44

[45] Eyckmans J, Roberts SJ, Bolander J, Schrooten J, Chen CS, Luyten FP. Mapping calcium phosphate activated gene networks as a strategy for targeted osteoinduction of human progenitors. Biomaterials 2013;34:4612-21.

[46] Duchamp de Lageneste O, Julien A, Abou-Khalil R, Frangi G, Carvalho C, Cagnard $\mathrm{N}$, et al. Periosteum contains skeletal stem cells with high bone regenerative potential controlled by Periostin. Nat Commun 2018;9:773.

[47] Wang J, Koelbl J, Boddupalli A, Yao Z, Bratlie KM, Schneider IC. Transfer of assembled collagen fibrils to flexible substrates for mechanically tunable contact guidance cues. Integr Biol 2018;10(11):705-18.

48] Fang F, Chew K, Chen X, Matsudaira P. Coactivators p300 and CBP maintain the identity of mouse embryonic stem cells by mediating long-range chromatin structure. Stem Cells 2014;32:1805-16.

[49] Tsai C-C, Hung S-C. Functional roles of pluripotency transcription factors in mesenchymal stem cells. Cell Cycle 2012;11:3711-2.

[50] Li L, Fan CM. A CREB-MPP7-AMOT regulatory axis controls muscle stem cell expansion and self-renewal competence. Cell Rep 2017;21(5):1253-66.

51] Siddappa R, Martens A, Doorn J, Leusink A, Olivo C, Licht R, et al, cAMP/PKA pathway activation in human mesenchymal stem cells in vitro results in robust bone formation in vivo. Proc Natl Acad Sci U S A 2008;105:7281-6.

[52] Doorn J, Siddappa R, van Blitterswijk CA, de Boer J. Forskolin enhances in vivo bone formation by human mesenchymal stromal cells. Tissue Eng Part A 2012;18:558-67.

[53] Gao B, Deng R, Chai Y, Chen H, Hu B, Wang X, et al. Macrophage-lineage TRAP+ cells recruit periosteum-derived cells for periosteal osteogenesis and regeneration. J Clin Invest 2019;129(6):2578-94.

[54] Bolander J, Chai YC, Geris L, Schrooten J, Roberts SJ, Luyten FP. Early BMP, Wnt and Ca2+/PKC pathway activation predicts the bone forming capacity of periostea cells in combination with calcium phosphates. Biomaterials 2016;86:106-18.

[55] Pauklin S, Vallier L. Activin/nodal signalling in stem cells. Development 2015;142 (4):607-19. 